\title{
The possibility of healing alveolar bone defects with simvastatin thermosensitive gel: in vitro/ in vivo evaluation
}

This article was published in the following Dove Press journal:

Drug Design, Development and Therapy

\section{Hong Ruan \\ Youcheng Yu \\ Xuehua Guo \\ Qian Jiang \\ Ying Luo}

Department of Stomatology,

Zhongshan Hospital, Fudan

University, Shanghai, China
Correspondence: Youcheng Yu

Department of Stomatology, Zhongshan Hospital, Fudan University, I80\# Fenlin

Road, Xuhui District, Shanghai, China

Tel +86 2l 64041990

Email yuyouchengdr@2Icn.com
Background: In this study, simvastatin (SVT) in situ gels were successfully produced by our group.

Methods: The preparations were characterized in the following aspects: in vitro gelation, drug release, stability and pharmacodynamics.

Results: In this study, drug content of prepared gels was found to be in the range between 89 and $92 \%$. The $\mathrm{pH}$ value was in the range between 6.5 and 7.0. The gelation temperature of the prepared thermogelling solutions was $37^{\circ} \mathrm{C}$. In vitro release showed that the release of SVT from in situ gels was slow with burst effects at an early stage. Researches indicated that intraorally slow release SVT in situ gels could effectively promote bone regeneration repair of alveolar bone defect.

Conclusion: This drug delivery system could prove to be a novel form able to prolong the residence time and to control the release of drug when administered into the oral cavity.

Keywords: SVT, in situ gel, thermogelling, gelation, in vitro release, alveolar bone defect

\section{Introduction}

It is well known that alveolar bone resorption in periodontitis is mediated by osteoclast cells, which are activated by several factors, including cytokines, that promote an increase in the receptor activator of nuclear factor k-B ligand (RANKL) in the osteoblast and favor RANR-RANKL linkage, which results in osteoclastogenesis and osteoclast activation. ${ }^{1}$ It reduces the bone height and width of implants, resulting in restoration problems and esthetic challenges in clinical dental treatment. Bioactive agents or bone growth factors, such as enamel matrix derivative, platelet-rich plasma, and platelet-derived growth factor, have been applied in conjunction with periodontal and alveolar bone regeneration. ${ }^{2-4}$ The authors previously demonstrated that three weekly local injections of simvastatin (SVT), ${ }^{5}$ a cholesterol-lowering drug with the capacity to induce bone morphogenetic protein- 2 and bone growth, ${ }^{6}$ resulted in a synergistic increase in new bones when applied to the periosteal surface of the rat mandible. ${ }^{7-10}$

Our previous studies also confirmed that the optimal concentration of SVT can improve the osteogenic activity of human periodontal ligament cells. ${ }^{11}$ However, it has been reported that $<5 \%$ of the drug can reach the systemic circulation due to extensive first-pass metabolism in the liver, and the clinical application of SVT available in the bone is confined by this liposolubility. ${ }^{12,13}$ Therefore, drug delivery systems of SVT should be designed to increase the aqueous solubility and improve the bioavailability.

Generally, biodegradable delivery system of natural polysaccharides have attracted much attention because of their good biocompatibility, biodegradability, and protective 
actions. ${ }^{14,15}$ Another interesting approach to oral drug delivery is the use of hydrogel systems. Hydrogels are hydrophilic polymers that can be swollen in water or water solvents. However, preformed hydrogels do not allow precise or reproducible administration of drugs, and after administration they often generate, in rapid clearance, unstable dose. Therefore, the most promising method is to use in situ formed hydrogels, which can be instilled as solutions and immediately gelled in contact with the mucous membrane. ${ }^{16}$

In situ gel, or environmental sensitive gel, is a new form of drug delivery in recent years. Different from the traditional formula, in situ gels are administered as low-viscosity solutions. In sensitive environment, the polymer changes conformation and produces gel, so it can not only prolong the contact time of the drug in the absorption part of the mouth but also slowly and continuously release the drug. Therefore, it is particularly useful for drugs that are used topically. Among in situ gels, the transition of phase can be caused by the change of temperature (thermosensitive) or the change of ionic strength (ion-sensitive). ${ }^{18}$

Poloxamer 407, polyoxyethylene-polyoxypropylenepolyoxyethylene triblock copolymer, is used for many pharmaceutical applications. The polyethylene oxide/ polypropylene oxide ratio of poloxamer 407 is 2 to 1 by weight. It is characterized by a sol-gel transition and shows thermoreversible gelation behavior according to the temperature. Hence, it can be easily applied and stays longer on the mucosal tissue. Moreover, the poloxamer systems can be easily administered by syringe equipped with needles appropriate for intrapocket delivery, becoming semisolid once in the periodontal pocket. ${ }^{19}$ If necessary, sol-gel transition temperature can be changed by adding salts to the solutions system to enable transition at body temperature.

Thus, the aim of this study is to develop an in situ gelling formulation of SVT based on Poloxamer 407 with respect to their concentrations in the simulated saliva. The preparations were studied in the following aspects: in vitro gelation, drug release, stability, and pharmacodynamics. The results suggested that the SVT delivered by in situ gel had a significant therapeutic efficacy in rat model. This strategy had a potential application in the repair of alveolar bone defect.

\section{Materials and methods Materials}

SVT was gifted by the Department of Pharmaceutics, School of Pharmacy, HuaDong University (Shanghai, China). Hydroxypropyl methylcellulose (HPMC K100M [80,000$1,20,000 \mathrm{cps}]$ ) and poloxamer 407 were obtained from
Colorcon Co., Ltd. (Shanghai, China) and BASF Chemical Company (Beijing, China), respectively. All other reagents were of commercially analytical grade and obtained from Sinopharm Chemical Reagent (Shanghai, China).

\section{Preparation of SVT gels}

The gels containing SVT for oral delivery were prepared in the lab of our group. For the preparation of $1.0 \%$ SVT gels, $20 \%$ poloxamer 407 and 2\% HPMC K100M were mixed with an adequate amount of distilled water in $100 \mathrm{~mL}$ volumetric flask, and the mixture was left in the refrigerator at $4^{\circ} \mathrm{C}$ for 24 hours. A preweighed amount of SVT was added to the abovementioned homogeneous solution and dissolved completely to obtain a homogeneous phase of polymer, solvent, and drug. Such a homogenization was performed using a lab stirrer at $1,300 \mathrm{rpm}$. A measured quantity of $0.1 \%$ methyl paraben (w/w, as preservative) was added to the preparation.

\section{Viscosity and rheological experiments}

The sol-gel transition temperature of copolymer sol in water was determined by the tube inversion method. ${ }^{20} \mathrm{~A}$ vial containing $20 \mathrm{~mL}$ copolymer sol was immersed in an oil bath at different setting temperatures and allowed to reach equilibrium. The sample was regarded as a "gel" when the flow rate was no longer visually observable within 30 seconds by inverting the vial, and the temperature was increased by $2^{\circ} \mathrm{C}$ per step. Then, the viscosity of the formulation, either in solution or in gel, was determined with a rotational viscometer (60 rpm, rotor 2$)$ using a proper sample $(20 \mathrm{~mL})$. Measurements were performed using suitable spindle numbers at different rotation rates. The viscosity was read directly from the viscometer display. Rheological experiments were performed using a cone and plate viscometer (HAAK, Vreden, Germany) at $37^{\circ} \mathrm{C}$. Approximately two to three drops of the sample were placed on the plate of the rheometer using a sampler, and the knob was released to bring the plate into contact with the tip of the cone. Measurements were carried out under the various shear rates, and the viscosity was read through the display device. All measurements were made in triplicate.

\section{Drug content}

A measured quantity of $1.0 \mathrm{~g}$ of the developed gels was dissolved in $100 \mathrm{~mL}$ phosphate buffer $(\mathrm{pH}=6.8)$ and left in the ultrasonic bath for 15 minutes, after which $10 \mu \mathrm{L}$ of the sample solution was withdrawn and diluted to $10 \mathrm{~mL}$. After the solution was filtered, the concentration of SVT was 
measured by the high-performance liquid chromatography (HPLC) method.

\section{Measurement of $\mathrm{pH}$}

In each formulated batch, $\mathrm{pH}$ was measured using the $\mathrm{pH}$ meter previously calibrated using standard buffers of $\mathrm{pH} 4$ and $\mathrm{pH} 7$ as per the established procedure.

\section{Stability studies}

Selected oral in situ gels were stored at room temperature for 3 months. After 1, 2, and 3 months, the $\mathrm{pH}$, gelling capacity, and drug content of the formulations were evaluated.

\section{In vitro drug release}

The release profile of SVT from the gels was evaluated using a dynamic dialysis method. ${ }^{21}$ Briefly, SVT in situ gels containing $10 \mathrm{mg}$ drug were placed into dialysis bags, which were then placed into an end-sealed vial. The release medium was $18 \mathrm{~mL}$ PBS ( $\mathrm{pH}=6.8$, containing $0.5 \%$ sodium dodecyl sulfate). The sealed vials were maintained at $37^{\circ} \mathrm{C}$ and $75 \mathrm{rpm}$ in a gas bath thermostatic oscillator. Aliquots $(1 \mathrm{~mL})$ were withdrawn at intervals of $0.25,0.5,1,2,4,6,8,12,16$, and 24 hours, and the same replacement volume was supplied as the release medium. The collected samples were injected directly into the HPLC system for analysis (wavelength=238 nm). An equal amount of free SVT was used for comparison.

\section{Pharmacodynamic evaluation}

\section{Animals}

All in vivo experimental protocols were approved by the animal care committee of the Faculty of Medicine, Fudan University Animal Center. In all studies of animals, the procedures were in line with the ethical standards of the agency and/or national research council, as well as the 1964 Helsinki declaration and its revised or comparable ethical standards. Sprague Dawley (SD) rats were used to investigate the pharmacodynamic effect of SVT in situ gel in this study. The weight of rats in each group ranged from 0.20 to $0.22 \mathrm{~kg}$, and all were placed in a well-ventilated room. The temperature was maintained at $20^{\circ} \mathrm{C} \pm 2^{\circ} \mathrm{C}$, with a relative humidity of $50 \%-10 \%$. Throughout the study, the animals were free to drink water.

\section{Experimental procedures}

Seventy-two 12-week-old SD rats were randomly divided into four groups: blank in situ gels (A), SVT solution (B), SVT gels (C), and SVT in situ gels (D) ( $\mathrm{n}=12$ in each group).
The blank in situ gel was used as the control. Rats were intravenously injected with $1 \%$ sodium pentobarbital at $3.2 \mu \mathrm{L} / \mathrm{g}$ before anesthesia.

A $2.3 \mathrm{~mm}$ diameter round carbide bur was used to prepare the alveolar bone defects in the mesial-lingual side of the left maxillary first molar mesiolingual root in each rat, and the size of each defect was ensured to be same as the round bur. Alveolar bone defects in the maxilla should be formed slowly and intermittently during drilling to prevent alveolar bone necrosis caused by excessive heat produced during the preparation of the defects. The SVT solution, SVT gels, SVT in situ gels, and blank in situ gels were lifted up by a scraper and then implanted into the defects in maxillary alveolar bone. After implantation, the mucosa was carefully sealed to avoid leakage of cell sheets. The animals were sacrificed at 14 and 56 days after operation, respectively, in the three experimental groups and the control group $(n=6)$, and the maxillae were prepared for micro-computed tomography (CT) analysis and histological staining to evaluate the effect of the repair of alveolar bone defects.

\section{Statistical analysis}

Data were statistically described in terms of mean \pm standard deviation. Student's $t$-test was used to evaluate associations between two groups' data. All statistics $<0.05$ were considered statistically significant. All statistical calculations were done using computer programs SPSS (Statistical Package for the Social Science; SPSS Inc., Chicago, IL, USA) version 19 for Microsoft Windows.

\section{Results \\ Characteristic of in situ gel}

In this study, drug content of prepared gels was found to be in the range between $89 \%$ and $92 \%$. The $\mathrm{pH}$ value of the optimized formulation was in the range between 6.5 and 7.0. An acceptable oral thermogelling gels must have a gelation temperature in the range of $36^{\circ} \mathrm{C}-37^{\circ} \mathrm{C}$ so as to be in liquid form at room temperature and to form a gel phase instantly in oral cavity. The developed formulations met all the prerequisites to perform an in situ gelling system and behaved like a fluid, but formed a rigid gel when at the temperature conditions of the oral cavity (Figure 1). In order to investigate the effect of the temperature during the gelation time, the fluidity of gels was measured using the viscosity method. The results of viscosity measurement showed that the preparations were typically of pseudoplastic systems and showed a marked increase in viscosity as temperature increased and 


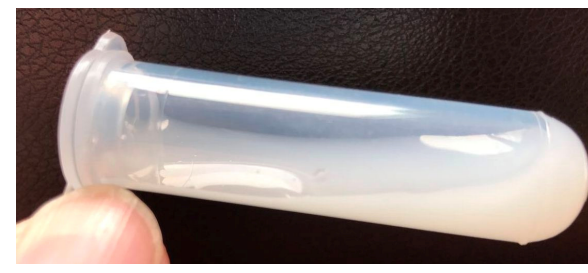

Before

Figure I Photographs of in situ gels formed before $\left(4^{\circ} \mathrm{C}\right)$ and after $\left(37^{\circ} \mathrm{C}\right)$ gelation.

ultimately formed a rigid gel. The gelation temperature of the prepared thermogelling gels was $37^{\circ} \mathrm{C}$ (Figure 2). The effect of shear rate on viscosity of the SVT in situ gels at $37^{\circ} \mathrm{C}$ was investigated. As shown in Figure 2, the gels' viscosity was markedly decreased when the shear rate was increased, and then it flattened. At high shear rate values, the polymer chains were no longer in a free-stretched state, suggesting a shear thinning behavior of the gels fluid. These results suggested that SVT in situ gels showed a non-Newtonian behavior, which was indicated by a higher viscosity at lower shear rates and lower viscosity at higher shear rates.

\section{Stability study}

SVT in situ gels exhibited good stability in the period of 3 months. There were negligible alterations in the initial values of viscosity of the formulations over the storage period. The samples were also analyzed for drug content by the HPLC method. The drug degraded to a negligible extent of $<5 \%$. No significant changes in the indexes and phase separation were observed during the observation period (Table 1).

\section{In vitro drug release}

The effect of polymer concentration on in vitro drug release from in situ gels is shown in Figure 3. The results showed

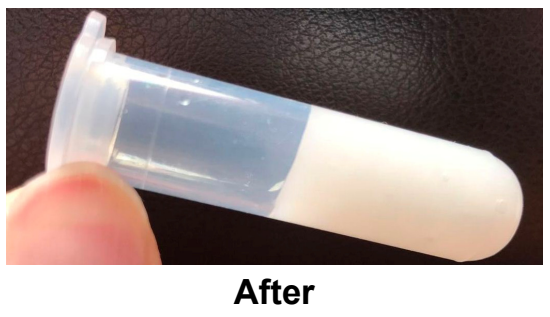

that the release of SVT from these gels was characterized by an initial phase of high release (burst effect). However, as the temperature increased, the hydrogel was formed and a portion of SVT might be loaded into the hydrogel phase. The remaining drug was released at a slower rate followed by a second phase of moderate release. This biphasic pattern of release is a characteristic of matrix diffusion kinetics. In addition, the release rate also depended on the polymer concentration (data not shown). As shown in Table 2, the in vitro drug-release kinetic model of SVT gels in PBS fits well with the Higuchi equation: $Q=9.172 t_{1 / 2}-1.291$ $(r=0.9991)$. Thus, it was speculated that the sustainedrelease property of in situ gels could enhance oral cavity absorption of SVT.

\section{Pharmacodynamic evaluation}

Animals were very calm after surgery, with free movement in cage. Around the teeth extraction socket, the gum and mucous membrane did not have inflammation or abnormalities. All rats can perform normal water intake 2 days after surgery. On the fifth day, periodontal dressings felloff around the teeth extraction socket surface and were completely covered by the epithelial tissue. The micro-CT analysis results of different formulations used in repairing of alveolar bone defects are
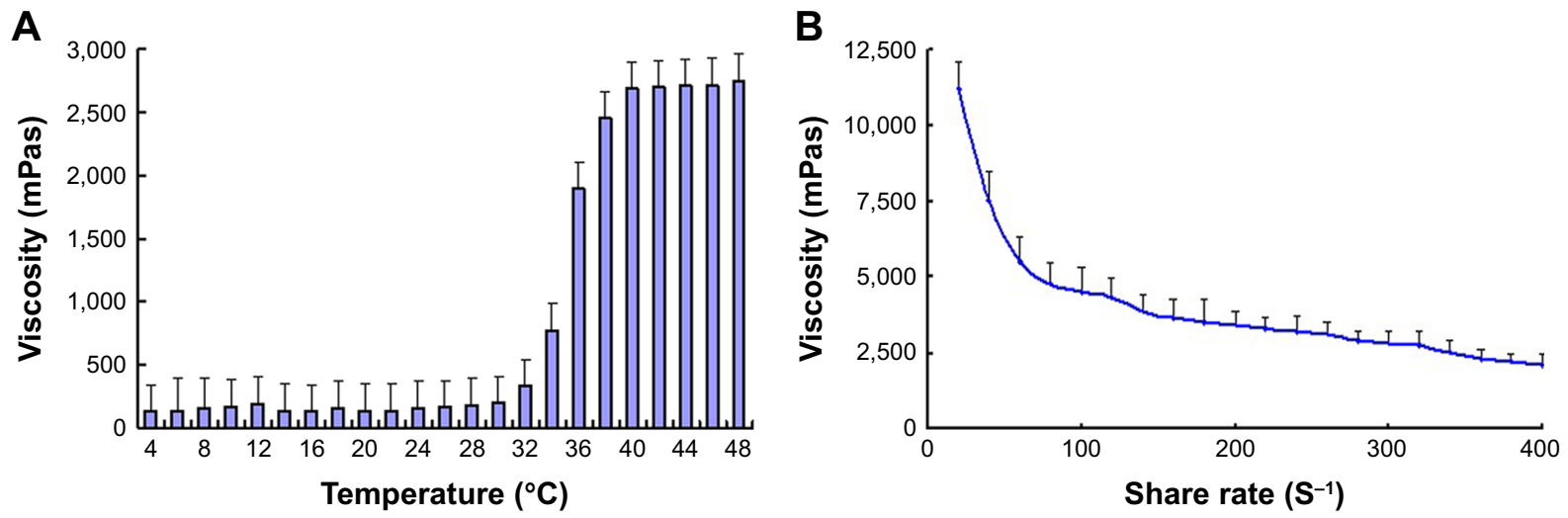

Figure 2 Mean viscosity-temperature trends profiles of SVT in situ gels. SVT (I.0\%, w/v), poloxamer $407(20 \%$, w/v), HPMC KI00M (2\%, w/v), and methyl paraben $(0.1 \%, w / v),(n=3)$.

Abbreviations: HPMC, hydroxypropyl methylcellulose; SVT, simvastatin. 
Table I The stability of SVT in situ gel during the observation period at the room temperature

\begin{tabular}{lllll}
\hline Date & Appearance & pH & Gelling capacity & Drug content (\%) \\
\hline 0 & Ivory & 6.61 & +++ & 91.8 \\
I month & Ivory & 6.56 & +++ & 90.9 \\
2 months & Ivory & 6.67 & +++ & 89.8 \\
3 months & Ivory & 6.71 & +++ & 87.5 \\
\hline
\end{tabular}

Abbreviation: SVT, simvastatin.

shown in Figure 4. At 14 and 56 days, the rats were sacrificed, and the maxillae were detected by micro-CT. Fourteen days after operation, the repair of the maxillary alveolar bone defect in rats was similar between the experimental group and the control group, with no significant difference $(P>0.05)$. After 56 days, the SVT solution or gel group had better repair of the maxillary alveolar bone defect than the control group (Figure 4 Group B and C). Meanwhile, micro-CT analysis indicated that the repair of the maxillary alveolar bone defect in rats with SVT in situ gels was better than that of the other groups (Figure 4 Group D). Further, the borders of the bone defects became indistinct and difficult to separate from the original alveolar bone. H\&E staining was performed to determine whether the SVT in situ gels group produced new bone compared with the other SVT formulation groups and the blank control group at 56 days (Figure 5). Notably, new bone trabeculae were relatively orderly, and the new bone tissue structure was similar to the normal bone tissue. Moreover, there were more vascular structures in the SVT in situ gels sheet group at 56 days.

\section{Discussion}

\section{Characteristic of in situ gel}

Statins were widely applied clinically to reduce cholesterol and for the treatment of hyperlipidemia and arteriosclerosis. In vivo and in vitro research found that statins activate osteoblast and promote bone formation. This research used sustainedly released in situ gel containing SVT as the functional delivery system to apply to the incisor teeth extraction socket. New bones formed during the alveolar groove healing process were evaluated for SVT in situ gels' enhancement effect on bone formation. With the changes of oral temperature, SVT in situ gel had formed gel in the teeth extraction socket. In this study, the carrier material used gradually degraded to glycolic acid and propionic acid, enter the tricarboxylic acid cycle, and eventually get discharged in $\mathrm{CO}_{2}$ and $\mathrm{H}_{2} \mathrm{O}$ formats. It did not influence bone metabolism and provided space for continuous growth of new bone tissues. On the basis of our previous study, aqueous solutions of poloxamer 407 were chosen as precursors of in situ-forming hydrogels. Combination products are novel medical products that combine drugs and devices. Compared with a single product, combination products, as a collection of single product functions, are more convenient to use; their effect is more significant and they have more potential advantages and development. With the development of modern medical technology, the type and quantity of combination products continue to increase. Its management often involves multiple regulatory departments to ensure that the development of the platform will not be greatly affected. In this study, SVT in situ gel is a typical

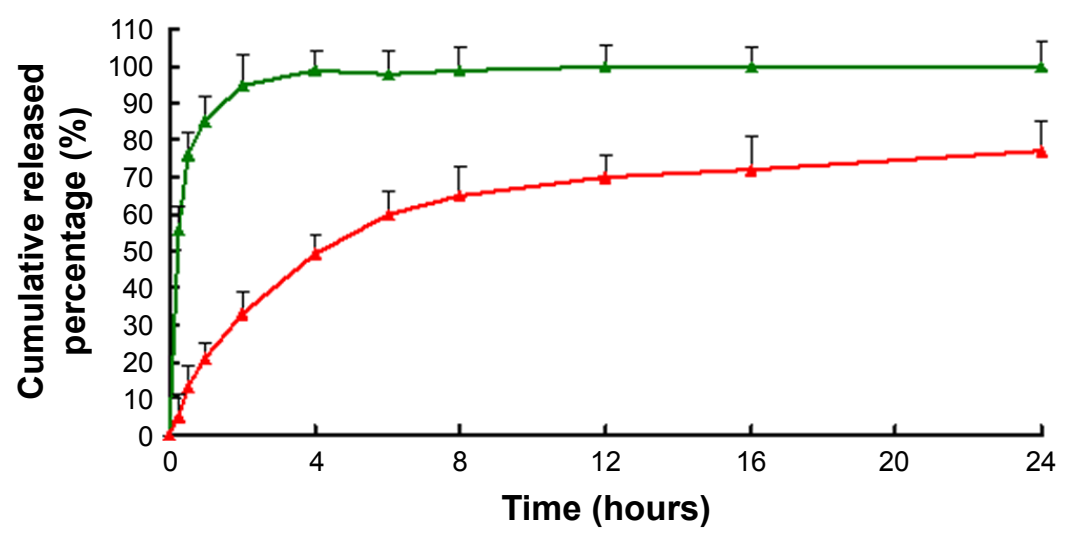

Figure 3 The in vitro drug-release profiles of SVT in situ gel and free SVT. Number represents the percentage of release ( $n=6$ ).

Note: Red curve represents in situ gel; green curve represents free SVT.

Abbreviation: SVT, simvastatin. 
Table 2 Correlation coefficients for kinetic analysis of release data for SVT gels

\begin{tabular}{llll}
\hline Formulation & \multicolumn{4}{l}{ Correlation coefficient $(\boldsymbol{r})$} \\
\cline { 2 - 4 } & Zero order & First order & Higuchi \\
\hline SVT gels & 0.8823 & 0.9321 & 0.9991 \\
\hline
\end{tabular}

Abbreviation: SVT, simvastatin.

combination product. If future clinic application is possible, more laws and regulations may be needed to further clarify the feasibility of the declaration.

\section{Pharmacodynamic evaluation}

Because micro-CT is a useful and reliable method for evaluating bone healing, in the current study, it was used for radiological analysis. According to the findings of micro-CT analysis, new bone formation in the maxillary alveolar bone defects increased over time and enhanced bone regeneration in the SVT in situ gels group compared with that in the other SVT groups and control group. According to this study, since the prepared in situ gels had a sustained-release pattern, the observed difference between the repair ability was remarkable. As mentioned earlier, this is mainly due to the prepared formulation expected to have an enhanced oral retention time due to an enhanced viscosity upon exposure to oral temperature. The gels will then be taken into the mucosa and they release the loaded drug for a longer time. However, in order to prove these animal conclusions, it is much desirable to study in vivo of humans.

\section{Conclusion}

In this study, SVT in situ gels were successfully prepared by our group. The drug content of the prepared gels was found to be in the range between $89 \%$ and $92 \%$. The $\mathrm{pH}$ value was in the range between 6.5 and 7.0. The gelation temperature of the prepared thermogelling solutions was $37^{\circ} \mathrm{C}$. In vitro release showed that the release of SVT from in situ gels was slow with burst effects at an early stage. Researches indicated that intraorally slowly released SVT in situ gels could effectively promote bone regeneration repair of alveolar bone defect. Therefore, this drug delivery system could prove to be a novel form able to prolong the residence time and to control the release of drug when administered into the oral cavity.
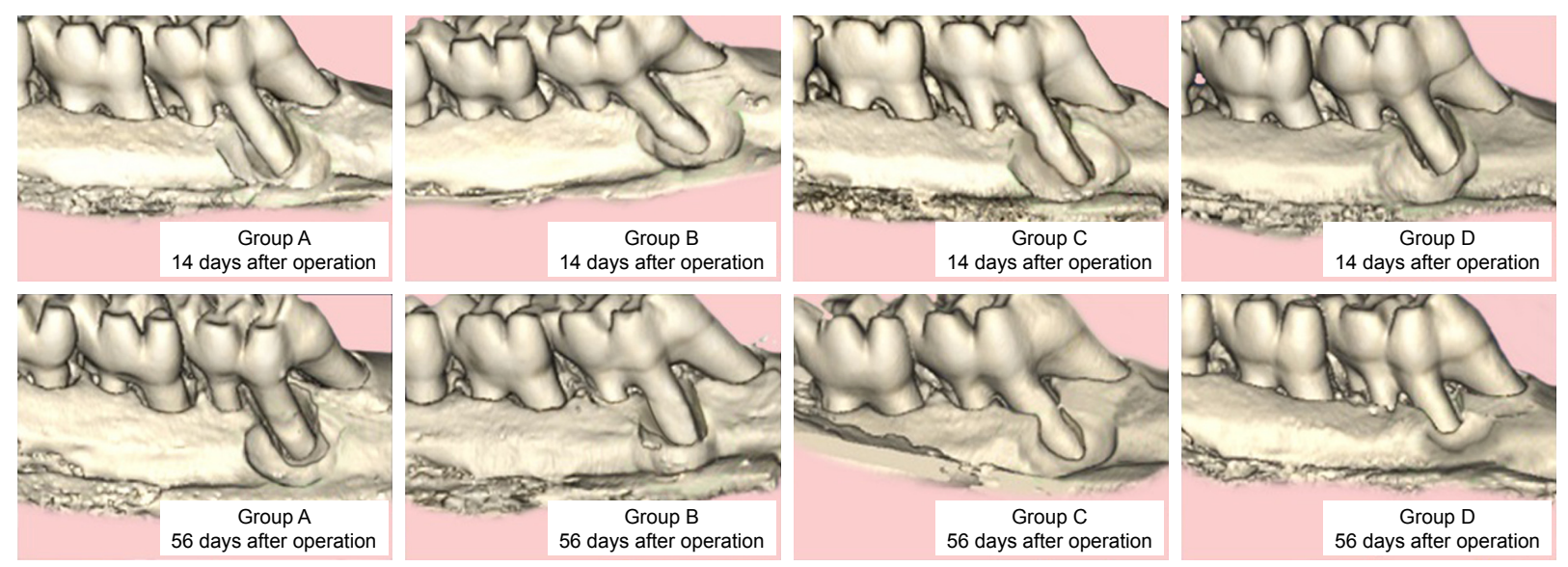

Figure 4 Bone density of teeth extraction socket of different SVT formulations in rats' model $(n=6)$. Abbreviation: SVT, simvastatin.

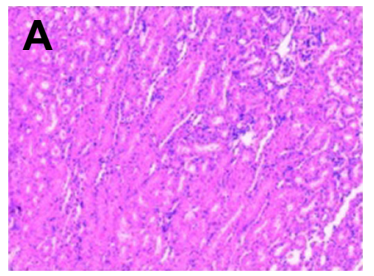

Blank in situ gels

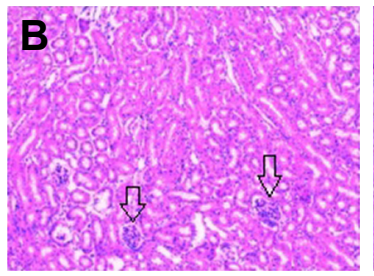

SVT solution

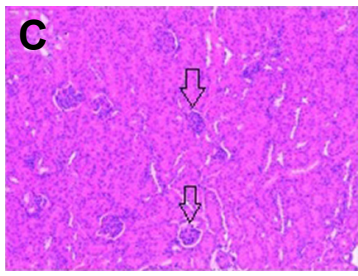

SVT gels

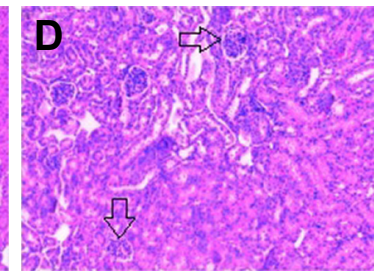

SVT in situ gels

Figure 5 After surgery, mandibular specimens were analyzed by histology. All tissue sections were isolated on 56 days after surgery and stained with hematoxylin and eosin. There are no significant differences among the four groups: (A) blank in situ gels, (B) SVT solution, (C) SVT gels, and (D) SVT in situ gels (I00× objective).

Note: The arrows refer to the inflammatory cells.

Abbreviation: SVT, simvastatin. 


\section{Acknowledgments}

The authors gratefully acknowledge funding support from the National Natural Science Foundation of China (Grant No 81670956), the State Key Program (Grant No 14JC1490600), and Shanghai Cooperative International Project (Grant No 16520710400) of Shanghai Committee of Science and Technology, China.

\section{Disclosure}

The authors report no conflicts of interest in this work.

\section{References}

1. Khajuria DK, Zahra SF, Razdan R. Effect of locally administered novel biodegradable chitosan based risedronate/zinc-hydroxyapatite intrapocket dental film on alveolar bone density in rat model of periodontitis. J Biomater Sci Polym Ed. 2018;29(1):74-91.

2. Khajuria DK, Patil ON, Karasik D, Razdan R. Development and evaluation of novel biodegradable chitosan based metformin intrapocket dental film for the management of periodontitis and alveolar bone loss in a rat model. Arch Oral Biol. 2018;85:120-129.

3. LiuY-S, Ou M-E, Liu H, et al. The effect of simvastatin on chemotactic capability of SDF- $1 \alpha$ and the promotion of bone regeneration. Biomaterials. 2014;35(15):4489-4498.

4. Steinhardt Y, Aslan H, Regev E, et al. Maxillofacial-derived stem cells regenerate critical mandibular bone defect. Tissue Eng Part A. 2008;14(11):1763-1773.

5. Mundy G, Garrett R, Harris S, et al. Stimulation of bone formation in vitro and in rodents by statins. Science. 1999;286(5446):1946-1949.

6. Golomb BA, Kane T, Dimsdale JE. Severe irritability associated with statin cholesterol-lowering drugs. QJM. 2004;97(4):229-235.

7. Dalcico R, de Menezes AM, Deocleciano OB, et al. Protective mechanisms of simvastatin in experimental periodontal disease. J Periodontol. 2013;84(8):1145-1157.

8. Liu YS, Ou ME, Liu H, et al. The effect of simvastatin on chemotactic capability of SDF- $1 \alpha$ and the promotion of bone regeneration. Biomaterials. 2014;35(15):4489-4498.
9. Seferos N, Pantopoulou A, Kotsiou A, Rallis G, Tesseromatis C. The influence of simvastatin in rats mandible and femur bone mass under Freund's adjuvant arthritis. Stomatologija. 2012;14(2):46-52.

10. Yin H, Shi ZG, Yu YS, Ys Y, et al. Protection against osteoporosis by statins is linked to a reduction of oxidative stress and restoration of nitric oxide formation in aged and ovariectomized rats. Eur J Pharmacol. 2012;674(2-3):200-206.

11. Hu F, Zhang XY, Wang CX, Zhou L. Effects of simvastatin on osteoblast activity of human periodontal ligament cells. Hua Xi Kou Qiang Yi Xue Za Zhi. 2009;27(3):313-316.

12. Yueyi C, Xiaoguang H, Jingying W, et al. Calvarial defect healing by recruitment of autogenous osteogenic stem cells using locally applied simvastatin. Biomaterials. 2013;34(37):9373-9380.

13. Zhang Y, Bradley AD, Wang D, Reinhardt RA, Statins RRA. Statins, bone metabolism and treatment of bone catabolic diseases. Pharmacol Res. 2014;88:53-61.

14. Ortega-Oller I, del Castillo-Santaella T, Padial-Molina M, GalindoMoreno P, Jódar-Reyes AB, Peula-García JM. Dual delivery nanosystem for biomolecules. Formulation, characterization, and in vitro release. Colloids Surf B Biointerfaces. 2017;159:586-595.

15. Liu G, Fang Z, Yuan M, et al. Biodegradable carriers for delivery of VEGF plasmid DNA for the treatment of critical limb ischemia. Front Pharmacol. 2017;8:528.

16. Maglione M, Spano S, Ruaro ME, et al. In vivo evaluation of chitosanglycerol gel scaffolds seeded with stem cells for full-thickness mandibular bone regeneration. J Oral Sci. 2017;59(2):225-232.

17. Li J, Gong C, Feng X, et al. Biodegradable thermosensitive hydrogel for SAHA and DDP delivery: therapeutic effects on oral squamous cell carcinoma xenografts. PLoS One. 2012;7(4):e33860.

18. Xu H, Shi M, Liu Y, Jiang J, Ma T. A novel in situ gel formulation of ranitidine for oral sustained delivery. Biomol Ther. 2014;22(2):161-165.

19. Esposito E, Carotta V, Scabbia A, et al. Comparative analysis of tetracycline-containing dental gels: poloxamer- and monoglyceridebased formulations. Int J Pharm. 1996;142(1):9-23.

20. Yu L, Zhang Z, Zhang H, Ding J. Biodegradability and biocompatibility of thermoreversible hydrogels formed from mixing a sol and a precipitate of block copolymers in water. Biomacromolecules. 2010;11(8): 2169-2178.

21. Liu X, Li X, Zhou L, et al. Effects of simvastatin-loaded polymeric micelles on human osteoblast-like MG-63 cells. Colloids Surf B Biointerfaces. 2013;102:420-427.

\section{Publish your work in this journal}

Drug Design, Development and Therapy is an international, peerreviewed open-access journal that spans the spectrum of drug design and development through to clinical applications. Clinical outcomes, patient safety, and programs for the development and effective, safe, and sustained use of medicines are the features of the journal, which has also been accepted for indexing on PubMed Central. The manuscript management system is completely online and includes a very quick and fair peer-review system, which is all easy to use. Visit http://www.dovepress.com/testimonials.php to read real quotes from published authors. 